\section{Consideraciones éticas en contexto de triage por COVID-19}

\section{Ethical considerations in the context of triage by COVID-19}

\section{Señor Editor:}

La actual situación de pandemia producto del coronavirus COVID-19 pone al sistema de salud ante una serie de desafíos éticos. Particularmente importante es lograr asegurar que quienes necesiten de cuidados intensivos (UCI), que se calcula ronde entre $5 \%$ y $16 \%$ de los pacientes COVID- $19^{5}$, lo obtengan a tiempo. Si se considera, como señaló José Miguel Bernucci, secretario general del Colegio Médico, que "en los últimos 5 o 6 días, la tasa de aumento de ocupación de camas críticas en promedio va en 20\%-30\%, cada 3 a 4 días se está doblando la cantidad de pacientes ventilados en Chile", la preocupación por contar con suficientes recursos no es exagerada.

En la actualidad contamos con dos lineamientos éticos nacionales, uno encabezado por en Centro de Bioética de la PUC y otro mandatado por la Mesa Social COVID-19 al rector de la misma Universidad, Ignacio Sánchez. Si bien los mencionados lineamientos constituyen un aporte en la difícil tarea de generar criterios que permitan orientar a los médicos en la priorización de pacientes a cuidados intensivos, las recomendaciones deben ser completadas con aspectos que aparecen como fundamentales en recomendaciones internacionales.

Este doble desafío, médico y ético, ha sido ya comenzado a ser discutido públicamente y consiste en anticiparse a la delicada situación de triage. Una estrategia del triage es necesaria cuando se constata que los recursos médicos disponibles son insuficientes para ofrecer tratamiento a todos los pacientes que se encuentren en riesgo vital, y los médicos deben clasificarlos en diversas categorías de importancia. Hay dos escenarios posibles en los que comparece el dilema ético del triage: a) caso de triage ex-ante: cuando se debe priorizar entre varios pacientes con riesgo vital que deben recibir atención y donde los recursos médicos son limitados; y b) caso de triage ex-post: cuando se debe determinar, si todos los equipos estén ya siendo usados por pacientes, en qué casos se debe continuar o cesar con los cuidados intensivos ${ }^{2}$. En ambos escenarios se trata de problemas éticos extremadamente complejos. En contextos de triage, de la forma que se decida priorizar, se beneficiará a algunos en desmedro de otros. Sin embargo, ante la catástrofe, como en una pandemia, la priorización busca maximizar los beneficios médicos. Esto último se entiende frecuentemente como salvar la mayor cantidad de vidas o al menos, ofrecer la mayor cantidad de cuidados médicos.
Los criterios éticos a los que debe ajustarse una estrategia de triage deben responder no solo a deberes de la ética médica, sino concordar con otros derechos más amplios. Así, toda estrategia de triage debe respetar el derecho de igualdad y la dignidad de las personas. En el contexto sanitario, significa asegurar que toda persona, independiente de su condición económica, género, etnia o edad, recibirá un trato igualitario y digno.

En los casos de triage ex-ante, un primer criterio médico fundamental para evaluar si un paciente debe ingresar al centro de cuidados intensivos es el pronóstico de beneficio clínico a corto plazo ${ }^{3,4}$. Aquí se trata de detectar al paciente que sacará el mayor provecho de la intervención en el más corto tiempo y priorizarlo. Desde luego esto implica también la evaluación de enfermedades crónicas y de la condición de salud general del paciente. Ahora bien, dado que la mayoría de los pacientes críticos por COVID-19 probablemente serán adultos mayores, es recomendable que los equipos médicos y sanitarios evalúen esta situación con especial sensibilidad ética.

En los casos de triage ex-post, es decir, en contextos donde pacientes se encuentran utilizando soportes vitales escasos y/o que se requieren con urgencia para otros pacientes críticos, el equipo médico debe evaluar constantemente la mejora terapéutica y/o estado de mejora de los pacientes. En casos de pacientes donde no se logre estabilizar al paciente o donde su estabilidad es de suma fragilidad y con una nula perspectiva de rehabilitación, no existe indicación médica para mantener los soportes vitales.

Un criterio fundamental en la ética médica y enfatizado transversalmente en diversas recomendaciones, es respetar la autonomía del paciente ${ }^{2}$. Ello significa aclarar, si el paciente ha dejado por escrito o expresado de otra forma, si deseaba ser reanimado y/o conectado a soportes vitales y bajo qué circunstancias. En caso de que el paciente no pueda expresarse en este aspecto, se precisa entonces de la información de terceros, como sus familiares, pero únicamente en virtud de aclarar la voluntad expresa del paciente. En ningún caso la voluntad del paciente debe ser supeditada a cualquier consideración de paternalismo médico o familiar.

El tercer criterio fundamental es el criterio del "control múltiple"1. Este criterio implica que las decisiones que se tomen con relación a la admisión o rechazo de pacientes a cuidados críticos deberá ser respaldada por otros profesionales de la salud. Cuando exista discrepancia, se recomienda delegar la decisión al Comité Ético Asistencial, quienes a su vez pueden guiarse por las recomendaciones éticas de la Comisión Asesora de Ética Asistencial del Ministerio de Salud (CEAM).

Desde luego, los criterios y las reflexiones presentadas en este texto no pretenden ser exhaustivos. No 
obstante, pretenden discutir acerca de los mínimos éticos necesarios para generar un marco ético referencial aceptable, capaz de apoyar la difícil labor que tendrán los profesionales de la salud en las salas de urgencia.

Dra. Diana Aurenque ${ }^{1}$ ${ }^{1}$ Universidad de Santiago de Chile (USACH), Santiago, Chile.

\section{Referencias}

1. Academia para Ética en Medicina; Akademie für Ethik in der Medizin (AEM) Possibilites and limits of institutional ethics services in response to the COVID-19 pandemic (english version): https://www.aem-online.de/fileadmin/ user_upload/AEM_Recommendations_Role_of_COVID-19_Pandemic_2020-03-31.pdf

2. Deutcher Ethikrat, Consejo de Ética Alemán, Recomendación por Corona-crisis: https://www.ethikrat.org/pressekonferenzen/der-deutsche-ethikrat-zur-corona-krise/

3. Recomendación de la Asociación alemana interdisciplinaria para medicina intensiva y de emergencia; Deutsche Interdisziplinäre Vereinigung für Intensiv- und Notfallmedizin
(DIVI) Entscheidungen über die Zuteilung von Ressourcen in der Notfall- und der Intensivmedizin im Kontext der COVID-19-Pandemie: https://www.aem-online.de/fileadmin/user_upload/COVID-19_Ethik_Empfehlung-v2.pdf

4. Recomendaciones de la Sociedad Suiza de Medicina Intensiva https://www.samw.ch/de/Ethik/Themen-A-bis-Z/ Intensivmedizin.html

5. Sociedades de Enfermedades Respiratorias, Epidemiología, Infectología y Medicina Intensiva. https://www.medicina-intensiva.cl/site/docs/sociedades_covid19.pdf

6. "Ventiladores mecánicos: El país está entre el 70\% y 80\% de capacidad de las máquinas, según intensivistas". La Tercera, 1.4.2020: https://www.latercera.com/la-tercera-pm/noticia/ventiladores-mecanicos-el-pais-esta-entre-el-70-y-80-de-capacidad-de-las-maquinas-segun-intensivistas/Z7RVJ666BRBADHHMUA7J6FNJNA/

Correspondencia a:

Dra. Diana Aurenque

Universidad de Santiago de Chile (USACH), Avda. Libertador Bernardo O'Higgins 3363, 9170019 Estación Central, Chile. Diana.aurenque@usach.cl 